\title{
Examining the Pathological Portions in MR Brain Slices using Automated Map and Improved Fuzzy K-Means Clustering
}

\author{
S.Vigneshwaran, Vishnuvarthanan Govindaraj, N.Anitha, M.Pallikonda Rajasekaran, \\ T.Arunprasath
}

\begin{abstract}
Identification of pathological structures (tissue and tumor region) in brain MR images is executed by an automated algorithm, and it requires improvement in processing time and segmentation accuracy. Oncological experts have predicaments in detecting the tumor masses that have similar resemblance with the tissue matters. An innovative amalgamation of soft computing algorithms, such as the automated map and clustering technique is presented through this paper. The Self-Organizing Map (SOM), a subsection of map technique, and the clustering process named the Improved Fuzzy K-Means clustering (IFKM) are used for the automated segmentation of MR brain structures in this paper. The segmentation outcomes of the algorithm are accurate for brain $M R$ image analysis, and it was evaluated using Jaccard index (TC), Mean Squared Error (MSE), Dice overlap Index (DOI) and Peak Signal to Nosie Ratio (PSNR) values in this paper. TC and DOI values were delivered as $84.43 \%$, $91.43 \%$, respectively. The efficiency of this algorithm is compared with other traditional approaches, and it has been confirmed that is better visualization of brain structures, which will greatly assist during Oncological treatment.
\end{abstract}

Keywords : Improved fuzzy k-means clustering, tumor identification, pathological detection, self-organizing map (som), MR brain image analysis.

\section{INTRODUCTION}

$\mathrm{S}_{\mathrm{e}}$ everal advancements of image segmentation process are mostly used in MR brain slices, which is useful to categorize the tumor and tissue regions. MR brain slices can be affected by some technical issues (parameter setting changing issue and noise influence), and mostly, the tumor portion cannot be easily reveled easily by a human instructor. Consecutively, this drawback can reduce the use of image segmentation algorithm. Recently, some researchers have analyzed the pathological portions using various soft computing

Revised Manuscript Received on December 16, 2019.

* Correspondence Author

Vigneshwaran*, Department of ECE, Kalasalingam Academy of Research and Education, Srivilliputhur, India. Email: a.s.vigneshwaran@gmail.com

Vishnuvarthanan, Department of BME, Kalasalingam Academy of Research and Education, Srivilliputhur, India. Email: gvvarthanan@gmail.com

Anitha, Department of ECE, Kalasalingam Academy of Research and Education, Srivilliputhur, India. Email: anithaa06@gmail.com

Pallikonda Rajasekaran, Department of ECE, Kalasalingam Academy of Research and Education, Srivilliputhur, India. Email: m.p.raja@klu.ac.in

Arunprasath, Department of BME, Kalasalingam Academy of Research and Education, Srivilliputhur, India. Email: arun.aklu@ gmail.com algorithms, which render accurate segmentation outcomes within minimum time. Tumor identification using Robust Fuzzy C-Means methodology proposed by Pham [1] needs improvement in processing time for providing ample segmentation outcomes. Ahmed et al. [2] recommended the modified FCM methodology which delivers better segmenting outcomes in more processing time. Karim et al. [3] proposed a methodology entitled Modified Fuzzy C Means that depends upon Gaussian Mixture Model, which could significantly assist in brain image segmentation process. This procedure delivers effective segmentation outcomes in more processing time. Karan et al. [4] proposed the Modified Fuzzy C - Means (MFCM) technique depending upon the histogram related cluster center assignment, which offers the ample segmentation outcomes, where more time is needed. A demarcation process of brain structures by Fuzzy Inference System technique proposed by Govindaraj et al. [5] delivers poorer PSNR and higher MSE values. Particle Swarm Optimization depending on Fuzzy C - Means approach suggested by Govindaraj et al. [6] delivers segmentation outcomes of brain MR slices with good segmentation accuracy. The automated mapping process depends upon the SOM neural network as suggested by Guler et al. [7], and it offers a demarcation of brain MR slices with minimum accuracy level. Demirhan et al. [8] recommended the combination of SOM and Leaning Vector Quantization algorithm, which needs human interface for effective segmentation outcomes. The combination of SOM and extended FCM approach proposed by Aghajari et al. [9] delivers the efficient segmentation outcomes in more processing time. Pathological identification process of brain MR slices analysis using SOM depends upon FKM algorithm and it was envisaged by Vishnuvarthanan et al. [10], where the algorithm demands more computational time for delivering significant segmented outcomes. The aforesaid interferences are determined by the new framework algorithm that has been developed in this paper.

The proposed methodology is clearly explained in section II, and the functioning of proposed methodology is illustrated in section III, and moreover, the completion of this process is discussed in section IV. 
Examining the Pathological Portions in MR Brain Slices using Automated Map and Improved Fuzzy K-Means Clustering

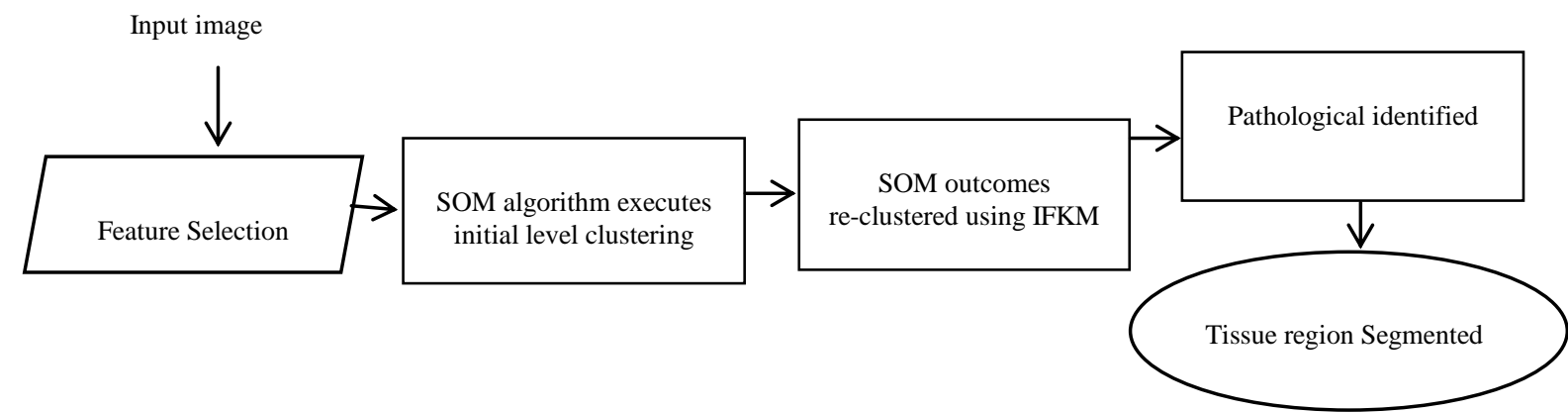

Fig. 1. Performance of the proposed SOM-IFKM algorithm

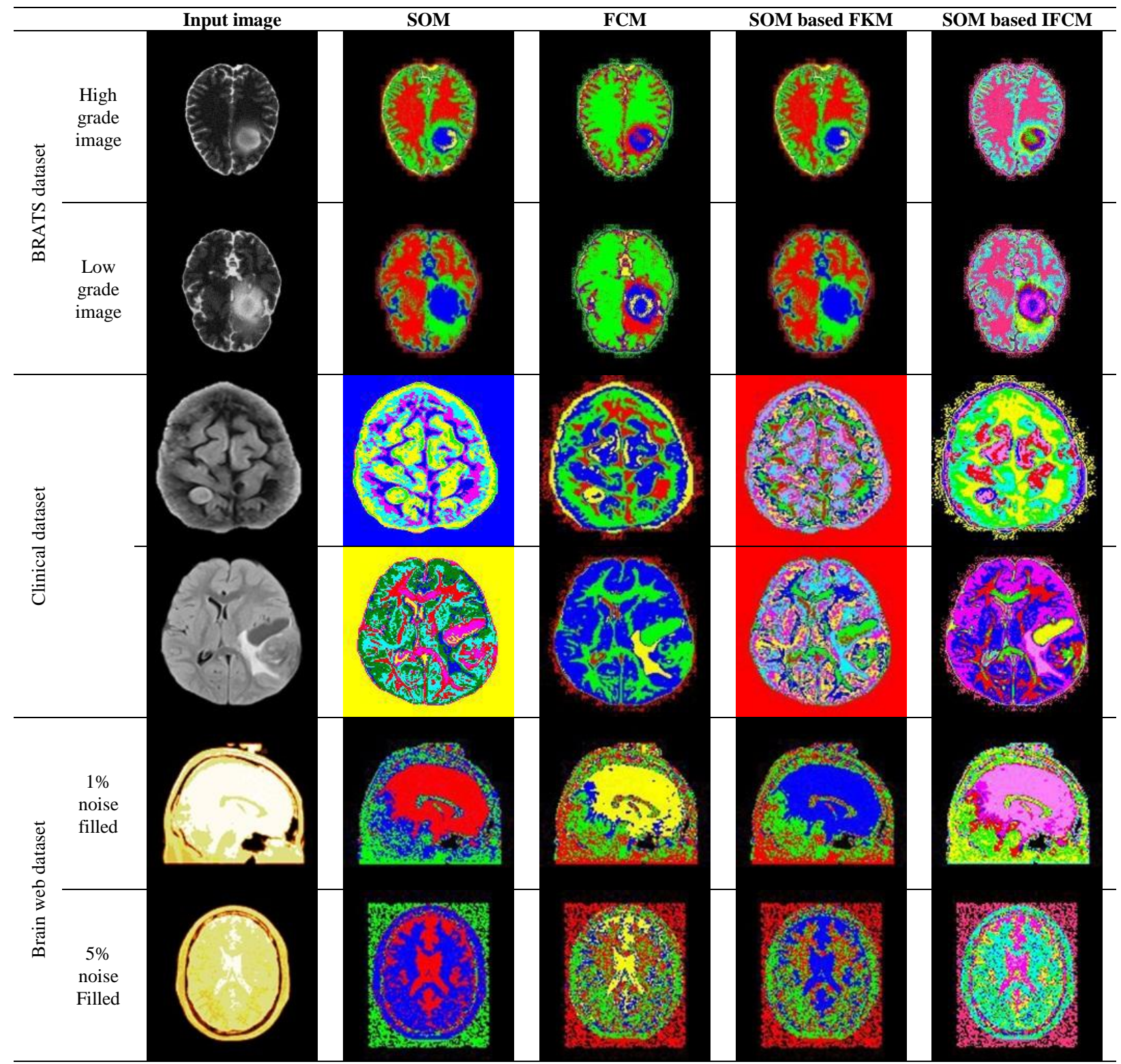

Fig. 2. Performance of the proposed SOM-IFKM algorithm

\section{PROPOSED METHODOLOGY}

Fig. 1 clearly explains the segmentation protocol of SOM based IFKM algorithm. SOM neural network is performed with the automated mapping process. The input of SOM algorithm is to get though the histogram based feature vector (mean and standard deviation of input image), and this recovers the over-fitting issue.

$$
\boldsymbol{D}_{\text {min }}(z)=\min \left\{\sum_{i}\left(x_{i}(z)-w_{i}(z)\right)^{2}\right\}
$$

Published By: equation (1). the dimensionality function with the aid of nearest neighbours and weight vectors. The nearest neighbours is obtained in

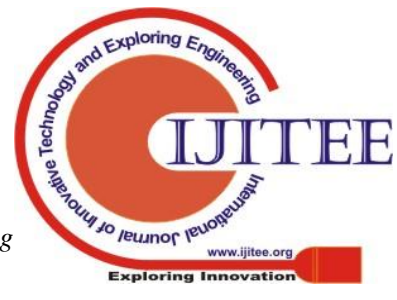


Table- I: Quality metrics analysis of the proposed algorithm

\begin{tabular}{|c|c|c|c|c|c|c|}
\hline \multirow{2}{*}{ Parameters } & \multirow{2}{*}{\multicolumn{2}{|c|}{ Clinical image }} & \multicolumn{2}{|c|}{ BRATS image } & \multicolumn{2}{|c|}{ Brain web database } \\
\hline & & & High & Low & & \\
\hline MSE & 0.0498 & 0.0549 & 0.0767 & 0.1511 & 0.0701 & 0.0862 \\
\hline PSNR & 61.1586 & 60.7378 & 59.2809 & 56.3395 & 59.6714 & 58.7776 \\
\hline Jaccard Index in $\%$ & 0.9261 & 0.9042 & 0.7509 & 0.8316 & 0.7747 & 0.8784 \\
\hline DOI values in $\%$ & 0.9617 & 0.9497 & 0.8577 & 0.9081 & 0.8731 & 0.9352 \\
\hline $\begin{array}{l}\text { Computational Time in } \\
\text { seconds }\end{array}$ & 5.9434 & 6.3046 & 6.9933 & 6.2976 & 6.1433 & 5.8183 \\
\hline $\begin{array}{l}\text { Memory requirement in } \\
\text { bytes }\end{array}$ & $2.20 \mathrm{e}+09$ & $2.24 \mathrm{e}+09$ & $2.20 \mathrm{e}+09$ & $2.18 \mathrm{e}+09$ & $2.21 \mathrm{e}+09$ & $2.20 \mathrm{e}+09$ \\
\hline
\end{tabular}

Table- II: Quality metrics obtained from various state-of-the-arts approaches

\begin{tabular}{|c|c|c|c|c|c|c|}
\hline Algorithm & MSE & $\begin{array}{c}\text { Memory } \\
\text { Requirement } \\
\text { in bytes }\end{array}$ & PSNR & $\begin{array}{c}\text { DOI } \\
\text { in bytes }\end{array}$ & $\begin{array}{c}\text { Jaccard Index } \\
\text { in bytes } \\
\text { in seconds }\end{array}$ \\
\hline SOM & 1.98 & $2.68 \mathrm{E}+13$ & 46.3817 & 80.89 & 67.91 & 68.1 \\
\hline FCM & 0.2731 & $5.20 \mathrm{E}+12$ & 53.7832 & 81.02 & & 2.0877 \\
\hline SOM based FKM & 2.421 & $1.45 \mathrm{E}+14$ & 43.8567 & 86.88 & & 76.69 \\
\hline
\end{tabular}

$$
w_{i}(z+1)=w_{i}(z)+\alpha(z) *\left(i-w_{i}(z)\right)
$$

The nearest neighbours are associated with Best Matching Unit (BMU), which is helpful for reducing the computation time of the segmentation process. Successfully, the SOM prototype $w_{i}$ is updated with the help of nearest neighbours and BMU. In equation (2), the SOM prototype is given as input to the IFKM algorithm. Then, the improved FKM algorithm is carried out through the entire information has to be obtaining from minimum Euclidean distance and cluster center, which can be updated iteratively. The Euclidean distance is evaluated by the nearest neighbour which means similarity pixels are connected to the cluster center. IFKM algorithm of objective function is given in equation (3), which provides the better segmentation outcomes using equations (4)-(8).

$$
J(U, M)=\sum_{i=1}^{K} \sum_{k=1}^{N} U_{k i}^{m} d_{k i}^{2}
$$

Let ' $d_{k i}$ ' represents Euclidean distance of $k^{\text {th }}$ pixel with respect to cluster ' $i$ '. ' $U$ ' refers to the updated membership function of $k^{\text {th }}$ pixel with respect to cluster $I$ (refers to the equation (5)).

$$
d_{k i}=\left\|w_{i}-v_{k}\right\|
$$

Where, ' $v_{k}$ ' refers to the cluster centroid (refers to the equation (8)) and ' $\left\|w_{i}-v_{k}\right\|$ ' represents the Euclidean distance for nearest neighbor in equation (4). The updated membership function is denoted as equation (6), which resolved with the aid of equation (7).

$$
\begin{aligned}
& U\left\{\begin{array}{l}
U_{k i} \in[0,1] \forall i, j \\
\sum_{i=1}^{K} U_{k i}=1 \forall k \\
0<\sum_{k=1}^{N} U_{k i}=1 \forall i
\end{array}\right. \\
& U_{k i}^{m}=\frac{\left(u_{k i}\right)^{m}}{1+\alpha} \\
& u_{k i}=\left[\left(d_{k i}\right)^{1 / m-1} \sum_{l=1}^{K}\left(\frac{1}{d_{i l}}\right)^{1 / m-1}\right]^{-1}
\end{aligned}
$$




$$
v_{k}=\frac{\sum_{k=1}^{N} U_{k i}^{m} x_{k}}{\sum_{k=1}^{N} U_{k i}^{m}}
$$

\section{RESULT AND DISCUSSION}

The functioning of proposed algorithm is extended to 112 input medical images that are received from clinical dataset, BRATS-2013 dataset and brain web dataset. The proposed framework proves the proficiency in case of segmentation outcomes, which is measured through quality metrics such Dice overlap Index, Jaccard index (TC), Peak Signal to Noise Ratio and Mean Squared Error [15]. The evaluated quality metrics are conveyed in Table I. Comparison of segmentation outcomes with SOM, FCM and the suggested algorithm is illustrated in Fig 2. Table II represents the SOM-IFKM methodology delivers superior demarcation accuracy when analysed with other traditional image segmentation algorithms.

\section{CONCLUSION}

An automated SOM depending on IFKM approach expounded by the authors utilized the segmentation process for pathological detection and identification. The SOM-IFKM methodology offers efficacious demarcation outcomes, which are then compared with FCM, SOM and SOM-FKM methodologies. The proposed SOM-IFKM methodology delivers superior PSNR values when compared with other image demarcation methodologies. Good segmentation outcomes are achieved by the proposed SOM-IFKM methodology, and it delivers higher DOI and Jaccard index values. Consequently, the recommended SOM-IFKM methodology works to offer accurate pathological identification and better visioning of the tumor portions. As an extension of this work, the performance of the proposed SOM-IFKM algorithm can be adjusted so as to get it applied towards volume rendering, and further archiving the same.

\section{ACKNOWLEDGMENT}

The authors thank the Department of Electronics and Communication Engineering of Kalasalingam Academy of Research and Education, Tamilnadu, India for permitting to use the computational facilities available in open source research laboratory. Also, the authors thank KGS Advanced MR \& CT Scan - Madurai, Tamilnadu, India, for supporting the research with patient information.

\section{REFERENCES}

1. D.L. Pham, "Spatial models for fuzzy clustering," Computer Vision and Image Understanding, vol. 84, 2001, pp. 285-297.

2. M.N. Ahmed, A.A Farag, N. Mohamed, T. Moriarty, and S.M. Yamany, "A modified fuzzy c-mean algorithm for basis field estimation and segmentation of MRI data," IEEE Transactions on Medical Imaging, vol. 21, 2002, pp. 193-199.

3. K. Karim, and M. Mohamed, "Image Segmentation by Gaussian Mixture Models and Modified FCM Algorithm," The International Arab Journal of Information Technology, vol. 11, 2014, pp. 11-17.
4. R. Karan, S. Nitesh, S.K. Pankaj, and K. Amith Mishra, "A fully automated algorithm under modified FCM framework for improved brain MR image segmentation," Magnetic Resonance Imaging, vol. 27, 2009, pp. 994-1004.

5. Govindaraj Vishnuvarthanan, Murugan Pallikonda Rajasekaran, "Segmentation of MR brain images for tumor extraction using fuzzy," Current Medical Imaging Reviews, vol. 9, 2013, pp. 2-6.

6. V. Govindaraj, and P.R. Murugan, “A complete automated algorithm for segmentation of tissues and identification of tumor region in T1, T2, and FLAIR brain images using optimization and clustering techniques," International Journal of Imaging Systems and Technology, vol. 24, 2014, pp. 313-325.

7. I. Guler, A. Demirhan, and R. Karakıs, "Interpretation of MR images using self-organizing maps and knowledge-based expert systems," Digital Signal Processing, vol. 19, 2009, pp. 668-677. DOI: https://doi.org/10.1016/j.dsp.2008.08.002.

8. A. Demirhan, M. Toru, and I. Guler, "Segmentation of tumor and edema along with healthy tissues of brain using wavelets and neural networks," IEEE Journal of Biomedical and Health Informatics, vol. 19, 2015, pp. 1451-1458. DOI: 10.1109/JBHI.2014.2360515

9. E. Aghajari, and G.D. Chandrashekhar, "Self-Organizing Map based Extended Fuzzy C-Means (SEEFC) algorithm for image segmentation," Applied Soft Computing, vol. 54, 2017, pp. 347-363. DOI: https://doi.org/10.1016/j.asoc.2017.01.003

10. G. Vishnuvarthanan, M.P. Rajasekaran, P. Subbaraj, and A. Vishnuvarthanan, "An unsupervised learning method with a clustering approach for tumor identification and tissue segmentation in magnetic resonance brain images," Applied Soft Computing, vol. 38, 2016, pp. 190-212. DOI: https://doi.org/10.1016/j.asoc.2015.09.016

11. J.C. Dunn, "A fuzzy relative of the ISODATA process and its use in detecting compact, well-separated clusters," J. Cybernetics, vol. 3, 1974, pp. 32-57.

12. J.C. Bezdek, "Pattern Recognition with Fuzzy Objective finction Algorithms," Plenum Press, New York, 1981.

13. Menze et al. "The Multimodal Brain Tumor Image Segmentation Benchmark (BRATS)," IEEE Trans. Med. Imaging, 2015.

14. D.L. Collins, A.P. Zijdenbos, V. Kollokian, J.G. Sled, N.J. Kabani, C.J. Holmes, and A.C. Evans, "Design and Construction of a Realistic Digital Brain Phantom," IEEE Transactions on Medical Imaging, vol. 17, 1998, pp. 463-468.

15. S. Vigneshwaran, Vishnuvarthanan Govindaraj, Pallikonda R. Murugan, Yudong Zhang, and Thiyagarajan Arun Prasath, "Unsupervised learning-based clustering approach for smart identification of pathologies and segmentation of tissues in brain magnetic resonance imaging," International Journal of Imaging Systems and Technology, 2019, pp. 1-18.

\section{AUTHORS PROFILE}

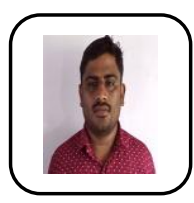

Vigneshwaran profile is a full time research schola in the Department of Electronics and Communication Engineering at Kalasalingam University. He has completed his M.E in Applied Electronics at Anna University in 2015 and B.E in Electronics and Communication Engineering at Sudharsan Engineering College in 2012, both located at Tamilnadu, India. His primary research focus is upon medical image processing.

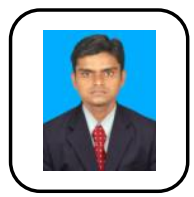

Vishnuvarthanan is an Associate Professor in the Department of Instrumentation Engineering at Kalasalingam University. Dr.Vishnuvarthanan received his Ph.D. in Electronics and Communication Engineering from Kalasalingam University, Tamilnadu in 2015 and completed his Bachelor of Engineering in Instrumentation and Control Engineering with first class in the year 2007 from Arulmigu Kalasalingam College of Engineering, Virudhunagar, Tamilnadu and Masters in VLSI with distinction in the year 2009 from Bharath University, Chennai, Tamilnadu. His areas of interest are Medical image processing and signal processing, and he has significant publications pertaining to these domains. 
Anitha has been awarded her $\mathrm{PhD}$ degree from Kalasalingam Academy of Research and Education (Kalasalingam University) in 2019 for her research work in the area of medical image processing. She received her Master's degree in Digital Communication and Networking in 2015 and Bachelor's degree in Electronics and Communication Engineering in 2006. She has three years of work experience as software programmer and two years of teaching experience, also an expertise in students' counselling. She is an active IEEE Member of Pothigai Subsection and IEEE Solid-State Circuits Society Member of IEEE Madras section from March 2018.

Pallikonda Rajasekaran is a Professor in Department of Electronics and Communication Engineering at Kalasalingam University. Dr. Pallikonda Rajasekaran received his Ph.D. in Electrical and Electronics Engineering from Anna University, Chennai in 2009, his M.Tech. in Biomedical Signal Processing and Instrumentation from SASTRA University in 2002 and his B.E. in Electronics and Instrumentation Engineering from Bharathidasan University (Shanmuga College of Engineering) in 2001. Dr. Pallikonda Rajasekaran research interests include biomedical instrumentation, image processing, wireless sensor networks, high performance computing, sensor systems, cloud computing, semantic networks. He has published numerous technical papers in refereed conferences and journals in these areas

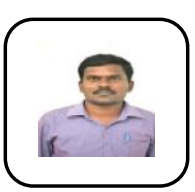

Arunprasath profile is an Associate Professor in the Department of Electrical and Electronics Engineering at Kalasalingam University. Dr. Arunprasath received his $\mathrm{Ph} . \mathrm{D}$. in Electronics and Communication Engineering from Kalasalingam University, Tamilnadu in 2015 and completed his Bachelor of Engineering in Electrical and Electronics Engineering with first class in the year 2006 from Anna University, Tamilnadu and Masters in Applied Electronics with distinction in the year 2009 from Anna University, Tamilnadu. His area of interest is Medical image processing, and he has appreciable publications in this area. 